\title{
A New Open Minimal Access Approach for Mesh Repair of Inguinal Hernia
}

\author{
M. Subrahmanyam, R. Sirisha, A. Deepthi, S. N. Mishra \\ Department of General Surgery, Kamineni Institute of Medical Sciences, Narketpally, T. S. India
}

Email address:

subrahmanyam_mutya@yahoo.com (M. Subrahmanyam),drsiri22@gmail.com (R. Sirisha), deepthia06@gmail.com (A. Deepthi), mutya2009@hotmail.com (S. N. Mishra)

\section{To cite this article:}

M. Subrahmanyam, R. Sirisha, A. Deepthi, S. N. Mishra. A New Open Minimal Access Approach for Mesh Repair of Inguinal Hernia. Advances in Surgical Sciences. Vol. 3, No. 4, 2015, pp. 27-31. doi: 10.11648/j.ass.20150304.11

\begin{abstract}
Mesh repair of inguinal hernia by open surgery is traditionally done with an oblique inguinal incision of 4-5 cm and by opening the inguinal canal by incising external oblique aponeurosis. We are presenting a new technique for mesh repair of inguinal hernia with two mini incisions, one over the superficial, and another over deep inguinal ring and without incising the external oblique aponeurosis. Methods: The study group comprised of 104 males patients undergoing surgery for inguinal hernia during the period January 2010- January 2015. Data regarding patient demographics, type of anesthesia given, operation performed, and complications were recorded. The operation was carried out under spinal or epidural anesthesia. With a transverse incision of size 1-1.5 $\mathrm{cm}$ at the superficial inguinal ring, the cord structures were reached and lifted up with the finger,. By passing an artery forceps with the tip upwards under neath the external oblique aponeurosis, another incision of 1$1.5 \mathrm{~cm}$ was made at the deep inguinal ring and the cord was lifted up by mobilizing. The indirect sac was dissected, ligated and mesh was sutured to the inguinal ligament by interrupted sutures and on the other side to conjoined tendon by retracting wound. The patients were followed up in the post-operative period. Results: There were 104 men with an age range of 20-64 years (mean35.6). On examination, 84 patients had indirect inguinal hernia and 20 patients had direct hernia. The incision size at superficial ring and deep rings measured at the end of the operation was $1.4 \mathrm{~cm}$, (range $1.2-2 \mathrm{~cm}$ ). Through the incision at the deep inguinal ring, the indirect sac could be identified, transfixation, ligation and excision of sac was done without difficulty. The mesh could be easily passed underneath the external oblique, spread and sutured. 3- sutures could be applied by retracting the external oblique. No drain was required in any repair. The mean operation rime was 54 minutes (range50-62 minutes).There was no post-operative hematoma or scrotal edema in any of the patients.. During a mean follow-up period of 48 months (range 12-60 months,), there was no recurrence and one patient had chronic pain. Conclusion: Inguinal hernia mesh repair with two mini incisions, one over the superficial inguinal ring and one over the deep inguinal ring and without incising the external oblique aponeurosis gives adequate exposure to place the mesh and repair the hernia. Follow- up did not show any recurrence or or significant chronic pain.
\end{abstract}

Keywords: Inguinal Hernia, Hernia Repair with Mesh, Minimal Access Hernia Repair

\section{Introduction}

The aim of surgical treatment of inguinal hernia is to reduce the rate of recurrence. One of the most important factors associated with rate of recurrence is anatomic structures in the region that remain under tension. The hernia recurrence rate is $1.4-22 \%$ in patients in whom repair is made with out the use of mesh. [1,2]

Repair of inguinal hernia with mesh was first reported by Usher in 1958 [3]. The technique has gained widespread acceptance due to its advantages like less tension and less pain which facilitates more rapid recovery and the rate of recurrence is lower as compared to other techniques. Many methods that utilize mesh via anterior and posterior approaches have been described. Lichtenstein, Stoppa, and Kugel frequently use such methods as laparoscopic extraperitoneal and intraperitoneal inguinal hernia repairs [47].Tension does not occur in hernias repaired using mesh and consequently the recurrence rate has reduced to less than $1 \%$.Risk of infection, rejection of mesh and chronic pain are other complications. The postoperative pain attributed to the repair of inguinal hernia with mesh is due to compression of the ilioinguinal or liohypogastic nerves between sutures of 
the mesh.

Lichtenstein procedure is the one which is commonly performed. The incidence of chronic pain following the surgery or inguinodynia varies from 6 to $9 \%$ in laparoscopic procedures and up to $33 \%$ in open surgeries [7].

Inguinal hernia mesh repair by open method is usually done with an oblique inguinal incision of $4-5 \mathrm{~cm}$. placed in the inguinal region, extending from pubic tubercle towards the anterior superior iliac spine. The external oblique aponeurosis is incised to approach the inguinal canal. A minimal trauma of access and preventive measures are likely to be helpful in reducing the complications. The concept of minimal access surgery has been practiced in many surgical specialities. By this analogy, a minimal access surgery in the mesh repair of inguinal hernia was studied. We present here a minimal access approach for hernia repair with mesh and assessed the results.

\section{Patients and Methods}

104 patients with inguinal hernia treated at General Hospital, Sangli and Kamineni Institute of Medical sciences Hospital, Narketpally, during the period 2010- 2015 formed the material for the study. Data regarding patient demographics, indications for surgery, type of anesthesia given, operation performed, complications were recorded. The exclusion criteria for this procedure were irreducible or partially irreducible hernia and recurrent hernia. Institute ethical committee have approved the study and informed consent has been taken from the patients.

The operative procedure was done under, spinal or epidural anesthesia. In supine position, the part was prepared and draped. The external inguinal 1 ring was palpated and a transverse incision was made over the ring measuring 1 $1.5 \mathrm{~cm}$.

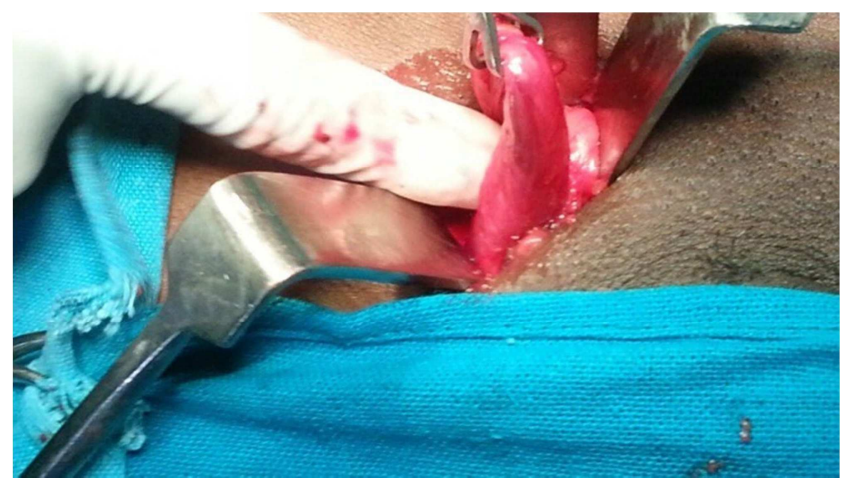

Fig. 1. Shows incision over external ring through which cord was lifted up.

Before the incision, a bolus dose of a second-generation cephalosporin was given intravenously. After incising the skin, subcutaneous tissue, through the external inguinal ring, the spermatic cord was elevated from the posterior wall of the inguinal canal [Fig. 1]. A finger was introduced underneath the external oblique aponeurosis, and after creating the space, an artery was passed and lifted up to about $4 \mathrm{~cm}$ and a transverse incision was made at that site measuring about $1-1.5 \mathrm{~cm}$. External oblique aponeurosis was divided to expose the cord structures. The cord was identified separated and lifted up. In cases of indirect hernias, the hernial sac was identified, dissected up to the internal ring and opened to allow examination of its contents. The sac was excised following high ligation and its distal portion was excised (Fig. 2).

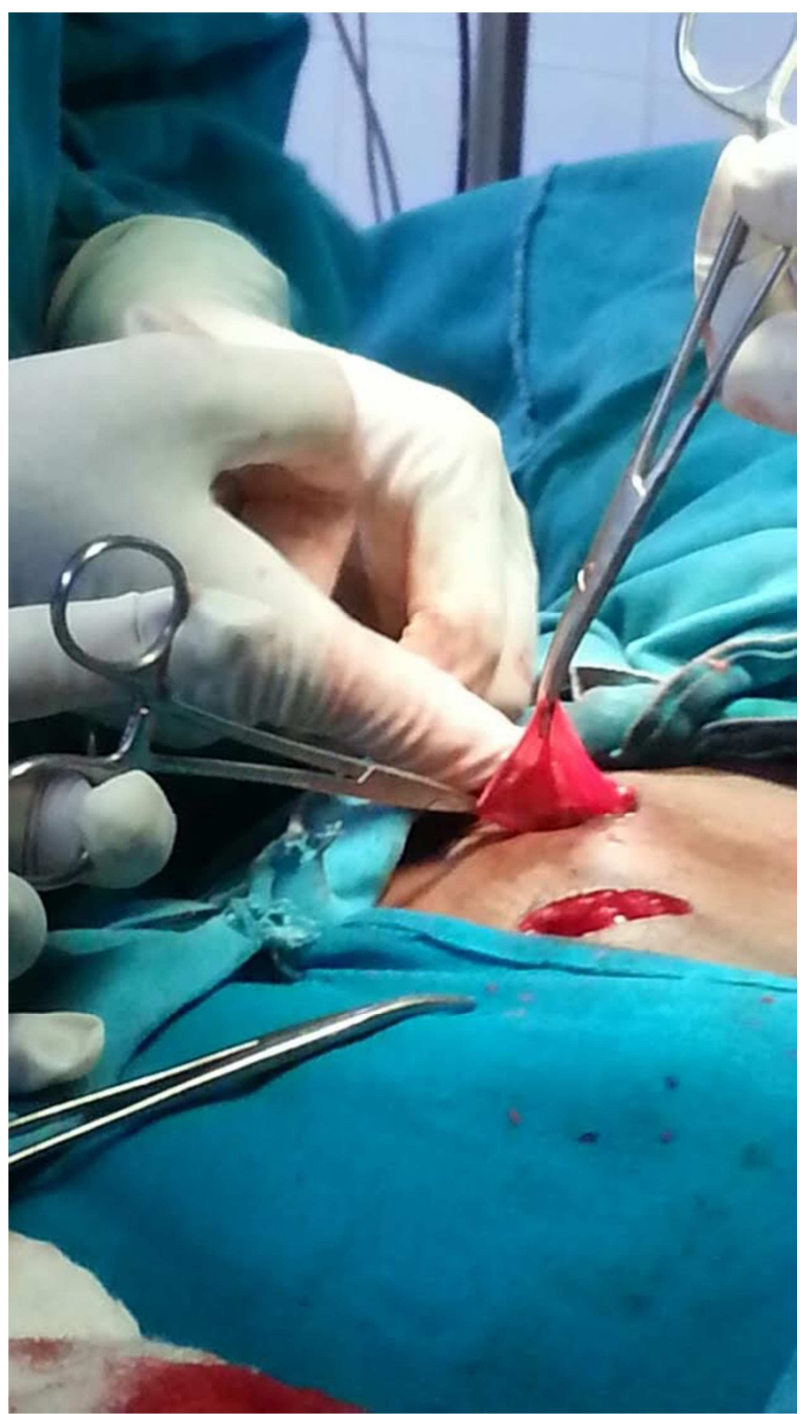

Fig. 2. Shows the two incisions made in this procedure and the indirect sac sac after dissection.

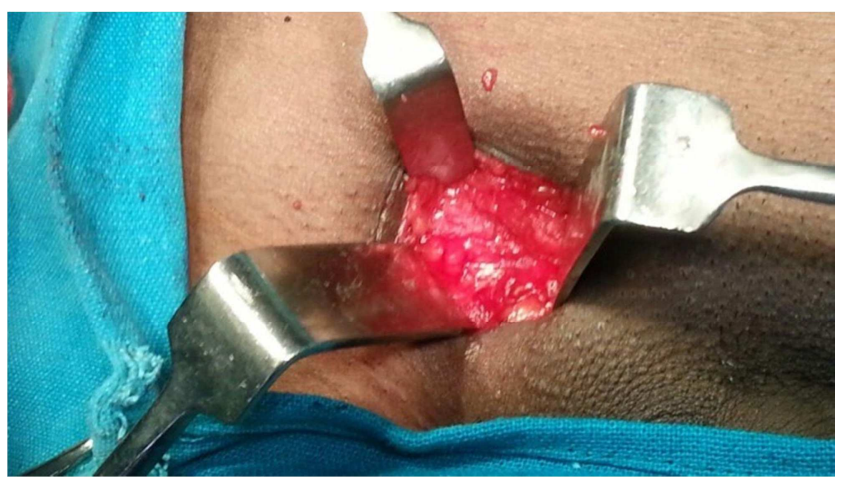

Fig. 3. Shows the mesh in position. 
However, in large indirect inguinal hernias, where the sac descents down to the scrotum, the distal part of the sac was left open to prevent the formation of a hydrocele, thus allowing spontaneous obliteration. In direct hernia, the sac was not opened, and was inverted with non-absorbable sutures (silk 2-0).

For both types of hernia, a polypropylene mesh (Proline, Ethicon Inc, $3 \times 5$ inch) was trimmed to fit the floor of the inguinal canal, and its apex was first sutured to the public tubercle using no 3-0 Proline sutures. The The lower border of the mesh was sutured to the free edge of the inguinal ligament, after an opening was made into its lower edge to accommodate the spermatic cord. In majority of repairs 3-4 interrupted sutures could be applied after retraction (Fig. 3) and sutures extended up just medial to the anterior superior iliac spine. Interrupted Proline sutures were used to suture the two cute edges of the mesh together around the spermatic cord above the internal inguinal ring. The infero-medial corner of the mesh is then attached well overlapping the pubic tubercle. The mesh was spread evenly without folding, and was then anchored to the conjoined tendon by interrupted sutures (Proline 3-0) after retracting the external aponeurosis,. After meticulous hemostasis, the wound was closed.

Regarding peri-operative care of the patient, prophylactic antibiosis was usually given for $48-72$ hours postoperatively. The patient was mobilized about six hours after surgery. Pain was measured as per visual analogue scale (0-10, 0 being nil and 10 maximum as per the patient. Postoperative analgesia was maintained with the administration of paracetamol or NSAIDS or a combination of these two analgesics. The duration of the hospitalization was recorded. When a closed suction drainage was used, it was removed at the time of discharge. The patients were followed up to look for pain, recurrence and other complications.

\section{Results}

There were 104 men with an age range of 20-64 years (mean35.6). 84 patients were found to be having indirect inguinal hernia and 20 patients had direct hernia. 74 patients were operated under spinal anesthesia and 30 under epidural anesthesia. None of the patients were operated under general, local or regional anesthesia. The incision size at superficial ring and deep rings measured at the end of the operation was $1.4 \mathrm{~cm}$, (range1.2 $-2 \mathrm{~cm}$ ). Through the incision at the deep inguinal ring, the indirect sac could be identified, transfixation, ligation and excision of sac was done without difficulty. In direct inguinal hernia, the sac was not opened and was inverted with non absorbable suture (3-0 silk.). The mesh could be easily passed underneath the external oblique, spread and sutured. 3- sutures could be applied by retracting the external oblique Fig. 1 - 3 show the incisions used, ligation of sac and mesh placement. No drain was required in any repair. The mean operation rime was 54 minutes (range50-62 minutes). There was no post-operative hematoma or scrotal edema in any of the patients. One patient had wound infection, which was treated by dressings. The mean hospital stay was 4.2 days(range 4-6 days).Pain in the postoperative period was 1-2 as per visual analogue scale. There was no hematoma or scrotal edema in any of the patients. One patient had wound infection which was teated by local dressings.

During a mean follow-up period of 48 months (range 126 months), there was no recurrence and one patient had chronic pain, which after treatment with analgesics, was relieved.

\section{Discussion}

Traditionally, inguinal hernia repair is being done by an oblique inguinal incision of 4-5 cms with or without mesh. This requires dividing the external oblique aponeurosis, which is one of the vital structures in preventing recurrence of hernia by sucking inside during the rise in intra abdominal pressure. Laparoscopic inguinal hernia mesh repair is popular, but it has not been established to be superior to the open method, in terns of time consumed, complications like recurrence and post operative pain.

Description of the Lichtenstein tension-free mesh repair opened a new era in groin hernia repair [6]. The traditional anterior inguinal hernia repair, where the inguinal canal is opened and the repair performed below the internal inguinal ring, has been utilized for decades with low hernia recurrence rates. With the advent of the Lichenstein, or tension-free, repair, which utilizes a biologically inert mesh to bolster the body's soft tissues rather than through rearrangement of the soft tissue itself, recurrence rates dropped even further. However, even with the advance associated with a tensionfree repair, the recovery associated with the anterior approach has typically been long and uncomfortable, traditionally incapacitating the patient for several weeks. More recently, a posterior approach, first described by Stoppa has also been used. In the posterior approach, the repair takes place in the preperitoneal space, above the internal inguinal ring, with the mesh material placed entirely within the space. A laparoscopic approach to hernia repair has been developed, modeled on the posterior approach; however, due to high reported rates of recurrence associated with this approach, as compared to traditional anterior approaches, it is usually reserved for treatment of recurrent hernias after an anterior repair[8-10]. Nevertheless, there is evidence to show that in experienced hands, posterior repairs of primary inguinal hernias have success rates approaching that of the anterior approach, with vastly improved postoperative recovery [1112]. However, the anterior approach method is simple, effective and is associated with a very low recurrence rates (ranging from 0 to $2 \%$ ) and can be performed under local or regional anesthesia. With these important advantages, it is currently the method for the plastic reconstruction of inguinal hernia for the majority of the surgeons around the world.

A variety of prosthetic mesh is available to the surgeon. The ideal mesh properties are inertness, resistance to 
infection, molecular permeability, pliability, transparency, mechanical integrity, and biocompatibility. Absorbable mesh does not remain in the wound long enough for adequate collagen to be deposited, while multi-filament mesh can harbor bacteria. Monofilament mesh is the most popular presently in use with the various types of polypropylene having different characteristic advantages [11]. Use of porous mesh (polypropylene) allows a large surface area for ingrowth of connective tissue leading to permanent fixation of the prosthesis within the abdominal wall. Intraparietal placement of the prosthesis allows well vascularized, tissue coverage of all aspects of the prosthesis. Fears of complications related to mesh implantation have proved to be without foundation. The use of vacuum drains is indicated in large inguinal hernias in order to minimize hematoma or seroma formation. However, duration of antibiotic use or indication for suction drainage differ among investigators [13-14]. In the present study, polypropylene mesh (monofilament) was used.

To reduce the chance of recurrence, the mesh should extent $2-4 \mathrm{~cm}$ beyond the boundary of Hesselbach's triangle [10]. The position of the mesh beneath the aponeurosis of the external oblique results in the intra abdominal pressure working in favor of the repair, since the external oblique aponeurosis keeps the mesh tightly in place by acting as an external support when intra abdominal pressure rises. In this procedure, the external oblique aponeurosis was not incised, it was only retracted and thus the the integrity was not disturbed. The mesh was fixed carefully, by the use of Prolene sutures and spread carefully to prevent folding, wrinkling. or curling of the mesh around the cord.

The method is simple, and has many advantages, such as effectiveness, safety, comfortable postoperative course with easily controlled pain, rapid return to unrestricted activities, an impressively low recurrence rate and high patient satisfaction. We have been encouraged by these good results of this procedure in, this study.

The proline mesh used to repair inguinal hernia in the present study is made of a monofilament poly-propylene that does not shrivel in the body due to its double knot structure. Moreover, it does not unravel when cut to fit a particular shape. It increases the strength of the inguinal canal, as a very strong fibrosis reaction occurs along and around the mesh when it is placed in the inguinal region. It has also been observed that in adults with indirect inguinal hernia, the use of mesh prevents the formation of a direct hernia later in life. Rejection of mesh, infection, seroma formation, edema of scrotum, orchitis are other complications. In our study infection occurred in 1 patient and no case of rejection or recurrence was noted during follow-up.

Inguinodynia or chronic postoperative groin pain following mesh repair is due to compression of the ilioinguinal or iliohypogastric nerve or genital branch of genitofemoral nerve between the sutures of the mesh. The pain may last more than 30 days after surgery and interfering with the patients activities of daily living or work activities. The incidence varies from 6-9\%. [14-17]. In the present study, only 1 patient, $(<1 \%)$ had chronic pain. It may be because of less tissue dissection and a few sutures used to fix the mesh.

Although numerous surgical approaches have been developed to treat inguinal hernias, the Lichtenstein tensionfree mesh-based repair remains the criterion standard $[8,9]$. Neverthless transabdominal preperitoneal (TAPP) or totally extraperitoneal (TEP) laparoscopic inguinal hernioplasty may offer specific benefits for some patients, such as those with recurrent hernia after conventional anterior open hernioplasty, those with bilateral hernias, and those undergoing laparoscopy for other clean operative procedures. [18, 19]. In view of so many options with varying results, we have tried this mini-incision approach. The main advantages are less dissection, keeping intact the external oblique aponeurosis, and less chances of inguinodynia.

\section{Conclusion}

Inguinal hernia mesh repair with two mini incisions, one over the superficial inguinal ring and one over the deep inguinal and without incising the external oblique aponeurosis give adequate exposure to place the mesh and repair the hernia. Follow- up did not show any recurrence or significant chronic pain.

\section{References}

[1] Rutkow JM, Robbins AW. Demoagraphic classificatory and socioeconomic aspects of hernia repairing the united states. Surg Clin North Am 1993; 73: 413-426.

[2] Welsh DRJ, Alexander MAJ. The Shouldice repair. Surg Clin North Am 1993;73:451-469

[3] Usher FC, Fries JC, Ochsner JL et al.. Marlex mesh a new plastic mesh for replacing tissue defects II. Arch Surg. 1959; 78: 138-145.

[4] Stoppa RE, Petit J, Henry X. Unsutured Dacron prosthesis in groin hernias. Int Surg. 1975; 60: 411-419.

[5] Lichtenstein IL, Shulman AG, Amid PK, et al. The tension free hernioplasty. Am J Surg. 1989; 157: 188-193.

[6] Popp LW. Endoscopic patch repair of inguinal hernia in a female patient. Surg Endosc. 1990; 4: 10-12.

[7] Ramshaw BJ, Tucker JG, Duncan TD. Laparoscopic herniorrhaphy: A review of 900 cases. Surg Endosc. 1996; 10: 255-232.

[8] Kurzer M, Belsham PA, Kark AE. The Lichtenstein repair. Surg Clin North Am. 1998; 78: 1025-1046.

[9] Amid PK, Shulman AG, Lichtenstein IL. Open "TensionFree" repair of inguinal hernias; The Lichtenstein technique. Eur J Surg. 1996; 162: 447-453.

[10] Goldstein HS. Selecting the right mesh. Hernia. 1999; 3: $23-$ 26.

[11] Amid PK, Shulman AG, Lichtenstein IL. Simultaneous repair of bilateral inguinal hernias under local anesthesia. Ann Surg. 1996; 223: 249-252. 
[12] Capozzi JA, Berkenfield JA, Cheaty JK. Repair of inguinal hernia in the adult with proline mesh. Surg Gynecol Obstet. 1988; 167: 124-128.

[13] Shulman AG, Amid PK, Lichtenstein IL. A survey of nonexpert surgeons using the open tension-free mesh repair for primary inguinal hernias. Int Surg. 1995; 80: 35-3.

[14] Aroori S, Spencer RA. Chronic pain after hernia surgery-an informed consent issue. Ulster Med J 2007; 76: 136-140.

[15] Poobalan AS, Bruce J, King PM et al. Chronic pain and quality of life following open inguinal hernia repair. Br J Surg 2001; 88: 122-126

[16] Staal E, Nienhuijs SW, Keemers-Geels ME et al. The impact of pain on daily activities following open mesh inguinal hernia repair. Hernia2008, 12: 153-157.

[17] Alfieri S, Amid PK, Campanell G et al. International guidelines for prevention and management of post-operative chronic pain following inguinal hernia surgery. Hernia 2011; 15: $239-249$.

[18] Lourenço A, da Costa RS. The ONSTEP inguinal hernia repair technique: initial clinical experience of 693 patients, in two institutions. Hernia. 2013; 17: 357-364.

[19] Myers E, Browne KM, Kavanagh DO et al Laparoscopic (TEP) versus Lichtenstein inguinal hernia repair: a comparison of quality-of-life outcomes. World J Surg. 2010; 34: 3059-64. 